Farum

Sociológico

\section{Forum Sociológico}

Série II

$27 \mid 2015$

Mobilidade científica \& imigração qualificada

\title{
Contratación internacional de médicos/as latinoamericanos/as en Portugal y España
}

Contratación internacional de médicos/as latinoamericanos/as en Portugal y España ${ }^{1}$

María Cristina Romero e Erika Masanet

\section{OpenEdition}

\section{Journals}

Edição electrónica

URL: https://journals.openedition.org/sociologico/1328

DOI: 10.4000/sociologico.1328

ISSN: 2182-7427

Editora

CICS.NOVA - Centro Interdisciplinar de Ciências Sociais da Universidade Nova de Lisboa

Edição impressa

Data de publição: 21 dezembro 2015

Paginação: 53-60

ISSN: 0872-8380

Refêrencia eletrónica

María Cristina Romero e Erika Masanet, «Contratación internacional de médicos/as latinoamericanos/ as en Portugal y España», Forum Sociológico [Online], 27 | 2015, posto online no dia 31 maio 2016, consultado o 29 março 2022. URL: http://journals.openedition.org/sociologico/1328 ; DOI: https:// doi.org/10.4000/sociologico.1328 


\title{
CONTRATACIÓN INTERNACIONAL DE MÉDICOS/AS LATINOAMERICANOS/AS EN PORTUGAL Y ESPAÑA ${ }^{1}$
}

\author{
María Cristina Romero \\ Universidad Complutense de Madrid, Facultad de Ciencias Políticas y Sociología, Departamento de Sociología I \\ Erika Masanet \\ Universidad de Valencia, Facultad de Ciencias Sociales, Departamento de Sociología y Antropología Social
}

\begin{abstract}
Resumo
A partir das experiências dos/as médicos/as latino-americanos/as que exercem a sua profissão em Portugal e em Espanha, o artigo busca comparar o recrutamento internacional dos/as médicos/ as, o reconhecimento das qualificações para o exercício da Medicina e os processos de integração profissional no sistema de saúde português e espanhol. Para isso, foi utilizada uma metodologia qualitativa baseada em entrevistas em profundidade. Os resultados mostram semelhanças e divergências em cada uma destas três dimensões nos dois contextos nacionais.
\end{abstract}

Palavras-chave: médicos/as latino-americanos/as, migração qualificada, recrutamento internacional

\begin{abstract}
From the experiences of Latin American doctors who practice their profession in Portugal and Spain, the article seeks to compare the international recruitment of doctors, recognition of qualifications for the practice of medicine and professional integration processes in the Portuguese and Spanish health system. For this, a qualitative methodology based on in-depth interviews was used. The results show similarities and differences in each of these three dimensions in these two national contexts.
\end{abstract}

Keywords: latin american doctors, skilled migration, international recruitment

\section{Resumen}

A partir de las experiencias de las/os médicas/os latinoamericanas/os que ejercen su profesión en Portugal y España, el artículo busca comparar el reclutamiento internacional de las/os médicas/os, el reconocimiento de las cualificaciones para el ejercicio de la Medicina, y los procesos de integración profesional en el sistema de salud portugués y español. Para tal fin, se utilizaron métodos cualitativos basados en entrevistas en profundidad. Los resultados muestran semejanzas y divergencias en cada una de las tres dimensiones en los dos contextos.

Palabras clave: médicos latinoamericanos; migración calificada; contratación internacional

\section{Introducción}

En la actualidad, los sistemas sanitarios para cubrir el déficit de personal médico pueden implementar principalmente cuatro estrategias: la formación interna, el mejoramiento de las condiciones de trabajo y flexibilización de la jubila- ción, el aumento de la productividad bajo parámetros de efectividad, y la contratación internacional de médicos/as en el extranjero (OCDE, 2008; EOHSP, 2006). Tanto la formación interna como la contratación internacional tienen un impacto directo en el stock de personal médico, sin embargo, debido a su inmediatez y bajo costo los sistemas 
sanitarios que cuentan con las condiciones socioeconómicas de atracción suficientes, hacen uso de la estrategia de la contratación internacional de médicos/as para cubrir las situaciones de escasez (OCDE, 2008).

Desde la década de 1990, Portugal ha recibido médicos/as extranjeros/as siendo entre 1998 y 2004 el periodo de mayor crecimiento. La entrada continua y creciente de estos/as profesionales a lo largo de las últimas dos décadas responde principalmente a una combinación de factores de expulsión y atracción, siendo la demanda de estos/as profesionales por parte del sistema sanitario, concretamente en determinadas especialidades médicas y regiones geográficas del país, un determinante significativo entre los pull factors. La demanda de médicos/as está relacionada principalmente con las asimetrías en su distribución geográfica (Alentejo y Algarve son las regiones con una importante escasez en todas las especialidades), y con el déficit de médicos/as en determinadas especialidades como Medicina General y Familiar, Obstetricia, Pediatría y Anestesia, siendo la mayor carencia en Medicina Familiar, así como el aumento de la demanda en el sector privado de la salud (Russo et al., 2012; Ribeiro et al., 2013).

En cuanto a España, se definen como estrategias principales de ingreso al mercado médico laboral el numerus clausus de las facultades y de la formación especializada, así como la contratación de médicos/as extranjeros/as para aliviar el déficit en este mercado a corto plazo (MSSSI y UPGC, 2011). No obstante, el problema radica en la ausencia de un sistema de información y de planificación coordinada de la fuerza médica laboral (Comisión Europea, 2010). En este sentido, la Organización Médica Colegial afirma que para el 2001 había un excedente de 15.000 médicos/as, en el 2007 un déficit de entre 15.000 y 20.000 , y desde el 2012 nuevamente un excedente de la misma cantidad de profesionales, si bien afirman que es prácticamente imposible saber si faltan o sobran médicos/as, o si tal vez, solo se necesitan en determinadas especialidades y en algunas zonas geográficas del país (OMC en Sevillano, 2014).

A partir de las experiencias de los/as médicos/as latinoamericanos/as que se encuentran ejerciendo legalmente su profesión en España y Portugal, el presente artículo tiene como objetivo comparar los mecanismos de contratación de estas/ os profesionales, el reconocimiento de credenciales para el ejercicio de la Medicina, y los procesos de integración profesional en los sistemas sanitarios español y portugués.

\section{Metodología}

Este artículo comparativo y descriptivo es el resultado de la contribución de dos proyectos de investigación desarrollados por las autoras sobre la migración de médicos/as latinoamericanos/as hacia Portugal y España, basados fundamentalmente en métodos cualitativos y que han utilizado como principal técnica cualitativa de recolección de datos la entrevista en profundidad. Concretamente, se llevaron a cabo entrevistas semiestructuradas a médicos/as latinoamericanos/as mediante un guión con las siguientes temáticas principales: aspectos biográficos y familiares, y trayectorias formativas, profesionales y migratorias. Los casos fueron elegidos mediante muestreo intencional orientado a recoger la diversidad de los discursos de la población entrevistada según el género, edad y país de procedencia.

Las entrevistas fueron grabadas en formato digital y posteriormente transcritas literal o parcialmente. Se realizó un análisis temático y descriptivo mediante la utilización del programa Sonar, en base a las siguientes categorías analíticas que responden al objetivo propuesto: mecanismos de contratación de los/as médicos/as, el proceso de reconocimiento de las cualificaciones para el ejercicio de la Medicina en el país de destino (reconocimiento del título de Medicina y de la especialidad, así como la obtención de la licencia de las organizaciones profesionales) y los procesos de integración profesional en los sistemas sanitarios de destino. De forma complementaria, también se llevó a cabo un análisis documental de diversas fuentes secundarias (legislación, reglamentación de las organizaciones profesionales y noticias relacionadas sobre el tema).

En Portugal, en el marco del proyecto "Skilled migration of health professionals in Portugal and Spain: a comparative study on the conditions of integration into the host society" se realizaron 30 entrevistas en profundidad a médicos/as generales y especialistas latinoamericanos/as que residen y trabajan en el país (11 mujeres y 19 hombres), cuyas edades oscilan entre los 27 y 62 años, y procedentes de Brasil, Uruguay, Cuba, Colombia, Venezuela, El Salvador y Costa Rica. De las 30 entrevistas, 15 se realizaron a médicos/as contratados/as en origen y las otras 15 a médicos que emigraron de forma independiente. Las entrevistas se realizaron principalmente en el Área Metropolitana de Lisboa, a excepción de una realizada en Oporto, entre febrero de 2011 y julio de 2012. Asimismo, se entrevistaron a 4 informantes-clave relacionados con la contratación en origen.

En España el análisis está basado en 52 entrevistas. Por una parte, en el marco de la tesis doctoral "Cartografías de profesionales de la medicina. Conversaciones con médicas-os latinoamericanas-os desde España", durante el año 2013 se entrevistaron a 36 médicos/as generales y especialistas de Latinoamérica ( 18 médicas y 18 médicos), con edades comprendidas entre los 25 y 63 años, entre los cuales una de las médicas fue contratada en origen. Los/as 
entrevistados/as son oriundos de Chile, Argentina, Uruguay, Cuba, México, Venezuela, Perú, Brasil, Ecuador, Colombia, República Dominicana, Paraguay, Bolivia, Nicaragua, y Guatemala, y residen en las comunidades autonómicas de Madrid, Cataluña, País Vasco, Valencia, Murcia, Aragón y Castilla-La Mancha. De otra parte, como parte del proyecto de Portugal mencionado anteriormente, se realizaron 16 entrevistas entre mayo y julio de 2011 a médicos/as generales y especialistas ( 7 mujeres y 9 hombres), una de ellas contratada en origen, procedentes de Brasil, Argentina, Colombia, Cuba, Venezuela y Uruguay, entre los 26 y 56 años, quienes residían en la Región Metropolitana de Barcelona y en Madrid. Dentro del mismo proyecto, se entrevistaron a dos informantes-clave relacionados con la contratación en origen en Cataluña.

\section{Contratación internacional de personal médico}

En el marco de la contratación internacional de personal médico por parte de los sistemas sanitarios de aquellos países que cuentan con los factores de atracción suficientes, a partir de los relatos de los/as médicos/as latinoamericanos/as que ejercen su profesión actualmente en España y Portugal es posible distinguir dos tipos de contratación, que a su vez, corresponden a dos perfiles diferenciados de estos/as profesionales: 1) reclutamiento en origen por las instituciones de los sistemas sanitarios; 2) migración autónoma y posterior contratación en el mercado laboral médico del país de destino.

\section{España}

La mayoría de los/as médicos/as latinoamericanos/as participantes en la investigación emigraron de forma independiente, bajo ningún acuerdo institucional en origen, y posteriormente fueron contratados/as en el mercado médico laboral español. De los relatos emergen diferentes motivos para iniciar un proyecto migratorio hacia España. Uno de ellos son las redes profesionales: "escogí España por comodidad, porque mis mejores amigos de carrera se venían para acá" (GEN-M, 29)², los vínculos familiares, y las relaciones afectivas. Asimismo, las complejas condiciones políticas, socioeconómicas y de inseguridad de la región actúan como factores de expulsión. Otra motivación muy importante es la realización de la especialidad en el sistema de formación español con las ventajas del estudio remunerado, la rapidez en los trámites: "en Estados Unidos son tres exámenes, el proceso es mucho más largo. No es como España que rindes el examen en enero, y en abril coges la plaza y ya empiezas en junio" (R1-H, 30), y la lengua común: "no pensé en Estados Unidos (...) estudié en un colegio público donde no tuve idiomas. Claro, si hubiera tenido la oportunidad me hubiera ido para allá, tengo compañeros allá (...) y también les pagan la residencia" (R3-H, 30). Y por último, las oportunidades reales de acceder al mercado laboral médico español:

Mi opción era irme para Inglaterra o para Estados Unidos. Pero aquí a pesar de que no era un programa de subespecialidad como en Estados Unidos, vi que me podía meter al sistema. No quería estar en un lugar donde me echaran directamente al haberla terminado y regresar a mi país. Yo quería tener un lugar donde terminando la estancia, existiera una posibilidad de quedarme. (ESP-H, 43)

De otra parte, si bien la contratación en origen no fue la tónica general de los/as médicos/as entrevistados/as, existen experiencias de este tipo hechas por instituciones sanitarias (públicas, privadas y mixtas) en algunas Comunidades Autónomas como Cataluña. Según informantes-clave entrevistados, estas ofertas de trabajo, de carácter puntual, han servido para cubrir las necesidades en determinadas especialidades y en los servicios de urgencias, y pueden ser de carácter genérico (proceso de selección realizado en el país de origen) o nominativo (dirigido a una persona en concreto). En este último caso, los relatos de los/as médicos/as entrevistados/as indican que la propia institución sanitaria les contrató a través de contactos y redes profesionales de algún amigo/a o colega que ya trabajaba en ese centro u hospital, así como les orientó y tramitó los permisos y licencias respectivos:

\footnotetext{
Mi amiga se vino y trajo mis papeles, me homologaron el título súper rápido, en seis meses. Al tiempo envié mi hoja de vida y en octubre del 2008 hubo una plaza aquí que se desocupó y el jefe de [especialidad] me escribió por correo para ofrecerme trabajo (...) decidí venirme y envié todos los papeles para que tramitaran el permiso de trabajo. (ESP-M, 40).
}

\section{Portugal}

El Ministerio de Salud Portugués llevó a cabo el reclutamiento internacional de médicos/as latinoamericanos/as a través de acuerdos bilaterales firmados con Uruguay (2008), Cuba (2009), Colombia y Costa Rica (2011), en respuesta a las necesidades de determinadas especialidades y regiones del país. En total fueron contratados/as aproximadamente 145 médicos/as en el ámbito de estos convenios: 12 de Uruguay, 40 de Cuba, 82 de Colombia y 9 de Costa Rica. En todos los casos la duración de los contratos de trabajo fue de tres años, con distintos años de inicio según los periodos de llegada 
de cada grupo. El reclutamiento se concentró en el área de atención primaria debido principalmente a las grandes carencias en este ámbito, a excepción de los médicos/as uruguayos/as que llegaron para trabajar en el Instituto Nacional de Emergencia Médica en Portugal (INEM) ${ }^{3}$.

En cuanto a aquellos/as médicos/as entrevistados/as que emigraron de forma autónoma, accedieron al mercado laboral médico a través de la Administración Pública y agencias privadas, una vez obtenidos el reconocimiento de sus cualificaciones y la regularización administrativa. Según las estadísticas del Ministerio de Salud portugués sobre recursos humanos extranjeros en el sector público sanitario, los/as médicos/as brasileños/ as conforman el segundo grupo mayoritario, sólo por detrás de los de España (Ministerio de Saúde, 2015). La importancia numérica de estos/ as médicos/as corresponde con la gran expresión migratoria que tiene, en general, este grupo nacional, cuya llegada empezó a ser fuertemente significativa durante la década de 1990. Esta migración responde principalmente a las vinculaciones históricas y culturales, siendo el factor lingüístico un elemento importante en esta elección (Masanet, 2012). De otra parte, el grupo de médicos/as cubanos/as entrevistados/as emigraron después de haber tenido una experiencia profesional en alguno de los Países Africanos de Lengua Portuguesa - PALOP (Angola, Mozambique, Sao Tomé e Príncipe, Guinea Bissau) a través de misiones de cooperación médica de Cuba. Esta experiencia marcó un antes y un después en sus vidas, y por motivos amorosos o ideológicos decidieron no regresar a su país una vez finalizada la misión de cooperación. Además, durante sus estancias en los países africanos establecieron contacto con portugueses que residían allí y que les incentivaron a emigrar por la demanda de médicos/as. Incluso en algunos casos, estas redes sociales les proporcionaron apoyo, facilitándoles información sobre el proceso de reconocimiento de la titulación y contactos para encontrar trabajo:

Tengo un amigo médico que está cerca de aquí (...) pero viven en Mozambique, entonces es socio de clínicas, y aquí viene de 15 en 15 días, entonces me resolvieron eso en Mozambique. Él es ingeniero en la construcción civil (...) gran amigo y entonces me resolvió un contrato de trabajo aquí, y entonces yo pedí visto de residencia con contrato de trabajo y así vine a Portugal, ellos me buscaron toda la documentación, me informaron de todo lo que tenía que hacer. (ESP-H, 44).

\section{Reconocimiento de credenciales} para el ejercicio de la Medicina

El reconocimiento de credenciales académicas que permiten el acceso al mercado médico laboral es un proceso fundamental, debido a la centralidad que la práctica de la Medicina tiene en el proyecto migratorio de estos/as profesionales. En este marco, la acreditación de títulos académicos en España y Portugal involucra dos procesos: 1) el reconocimiento de los títulos de carrera y de especialidad; 2) la obtención de la licencia en las diferentes asociaciones profesionales médicas.

España

Según la legislación española, el reconocimiento del título de Medicina está a cargo del Ministerio de Educación, Cultura y Deporte (MECD), y se realiza mediante la presentación de la documentación requerida. Los relatos de los/as médicos/as entrevistados/as coinciden en la facilidad para la obtención de la homologación de este título, pudiéndose tramitar inclusive antes de viajar o sin tener un proyecto claro de migración: "había sido precavido y había homologado mis estudios de medicina cuatro años antes de venirme" (ESP-H, 45).

No obstante, esta facilidad en la concesión de la homologación del título en Medicina contrasta con la dificultad del reconocimiento de la formación especialista. En mayo de 2010 fue aprobada la nueva legislación para el reconocimiento del título de especialista por parte del Ministerio de Sanidad, Servicios Sociales e Igualdad (MSSSI) obtenido en los estados no miembros de la Unión Europea (Decreto Real 459/2010, abril 16) ${ }^{4}$, cuya principal novedad fue proporcionar una alternativa a los/as especialistas sin acreditación quienes venían ejerciendo su especialidad. Sin embargo, a pesar de la disminución de barreras en relación con la anterior legislación de 1991, según estimaciones de ASOMEX (Asociación de Médicos Especialistas Extracomunitarios), el MSSSI ha rechazado entre el $80 \%$ y $90 \%$ de las solicitudes de especialistas no comunitarios/ as en los tres primeros años de implementación de la ley, lo que evidencia un alto número de resoluciones negativas ${ }^{5}$. Tendencia constatada con datos estadísticos suministrados por el MSSSI para esta investigación que confirman la dificultad del trámite de homologación del título de especialista frente al de carrera: de 1987 a 2012 se han homologado 57.533 títulos de Medicina ( $86 \%$ a médicos/as generales de Latinoamérica) frente a 3.001 de especialista (26\% a especialistas latinoamericanos/as). Asimismo, las experiencias de aquellos/as que han obtenido el reconocimiento de la especialidad también relatan las dificultades de este proceso: 
Nos presentamos 48 al examen y pasamos 4 (...) Era un examen que solamente te podías presentar 2 veces en la vida (...) Se homologa con un examen y además tienes que cumplir requisitos, mi especialidad en [país LAM] eran 4 años y aquí son 5 . Entonces tenía que haber demostrado que tenía 2 años de experiencia (...) Es la primera vez que un examen me iba a costar algo en la vida, porque era todo o nada. De hecho el examen escrito no lo pasé en la primera tanda, me quedé a 4 puntos (...) Entonces impugné el examen y pasé a la segunda ronda que era un examen oral que traían profesores de toda España. (ESP-H, 43).

Por último, junto al reconocimiento de los títulos de formación académica del nivel de Medicina y de especialidad, la legislación de España requiere la acreditación expedida por el Colegio de Médicos para ejercer la profesión en el país. Al respecto las narrativas expresan que, en general, este trámite administrativo de colegiación transcurre con bastante facilidad y rapidez para el caso de los/as médicos/as latinoamericanos/as entrevistados/as.

\section{Portugal}

Según legislación portuguesa, para los/as médicos/as extracomunitarios/as el reconocimiento del título de Medicina está gestionado por las Facultades de Medicina, y consiste en un examen de conocimientos médicos con una parte teórica y una práctica de resolución de casos clínicos, así como un prueba de lengua portuguesa si corresponde. En el caso de los/as médicos/as reclutados/as en origen en 2008, 2009 y 2011 (Cuba, Uruguay, Colombia y Costa Rica), de acuerdo con los informantes-clave y los/as médicos/as entrevistados/as, estos exámenes son los mismos realizados durante el proceso de selección en el país de origen haciendo el proceso, tanto de selección como de reconocimiento, más rápido y facilitado por las instituciones debido a las necesidades del país contratante. Situación muy diferente a la que relatan los/as médicos/as latinoamericanos/as entrevistados/as que migraron de forma autónoma, cuyos procesos de reconocimiento de este título tardaron entre uno y dos años, además de los altos costes generados por la traducción al portugués en caso de ser necesario.

De otra parte, los distintos Colegios de las Especialidades de la Organización Médica Portuguesa son los responsables del reconocimiento de la especialidad obtenida en el extranjero, con la particularidad de que el procedimiento es el mismo para nacionales y extranjeros/as. La reglamentación al respecto exige, en primer lugar, la presentación de un detallado Curriculum Vitae (CV) que de ser aprobado, permitirá presentarse el examen final de acceso al título de especialista (valoración del CV, más pruebas teóricas y prácticas). Si el CV no es aprobado, se deben realizar unas prácticas en la especialidad para llenar los vacíos y poder optar nuevamente al examen, que de acuerdo con las entrevistas, pueden variar de uno a tres años. Si bien no existen barreras institucionales específicas para los/as especialistas extranjeros/as, tal y como valoran los/as médicos/ as entrevistados/as, la evaluación del CV puede implicar una mayor desconfianza en la formación de la especialidad realizada en los países de origen, lo cual incrementa la probabilidad de una evaluación negativa con la necesidad de realizar prácticas, y el alargamiento del tiempo del proceso. Además de este elemento subjetivo, estos médicos/as también expresan que el menor tiempo de formación en algunas especialidades y la no existencia de otras, acarrean evaluaciones negativas: "hice el año común y después entré nuevamente en la especialidad porque mi especialidad no fue reconocida. Es que la geriatría no existe como especialidad en Portugal. (...). Entonces empecé a hacer la especialidad en salud pública para ver cómo era" (ESP-H, 47) ${ }^{6}$.

Por último, la Organización Médica Portuguesa establece un sistema complejo de acreditación profesional que distingue el ejercicio autónomo o independiente de la profesión del ejercicio tutelado. En el primer caso, se requiere de la comprobación de tres años de experiencia profesional en los últimos cinco años para los/as médicos/as extranjeros/as. Si no consiguen la licencia de Medicina Autónoma, los/as médicos/as deben realizar unas prácticas bajo la supervisión de un/a doctor/a, que en la mayoría de los casos corresponde a nueve meses de formación. Asimismo, es exigida la prueba de Comunicación Médica para aquellos/as médicos/as de lengua no portuguesa. En general, los/as médicos/ as latinoamericanos/as entrevistados/as no tuvieron problemas para conseguir la acreditación profesional y ejercer su profesión, salvo los/as médicos/as de Costa Rica y Uruguay contratados/as en origen.

\section{Dinámicas de integración profesional}

En aras de dar explicación a los procesos de integración profesional de los/as médicos/as latinoamericanos/as que actualmente ejercen su profesión en España y Portugal, seguiremos la clasificación de los dos perfiles descritos inicialmente al implicar cada uno diferentes condiciones de incorporación laboral: migración de los/as médicos/as en el ámbito de un acuerdo institucional, y migración autónoma o independiente de estos/as profesionales.

España

En cuanto a la contratación en origen, como se mencionó anteriormente, las instituciones con- 
tratantes proveen una oferta de trabajo así como la tramitación de los permisos y licencias, facilitando de este modo los procesos de incorporación laboral. En el caso de la migración autónoma, los procesos exitosos de integración al mercado médico laboral, público y privado, están en relación con la oferta disponible: "llegué en un momento que no había crisis, y fue más fácil insertarte laboralmente. Claro, con la predisposición que tenemos los latinos de trabajar mucho más" (ESP-H, 35), o por el contrario, por la dificultad en momentos de superávit de profesionales: "nadie la tiene fácil, ni el español ni el latino y menos si no tiene papeles" (R3-H, 28). Asimismo, para aquellos/as que no han podido homologar la especialidad se presentan situaciones de descualificación profesional: "he visto médicos que trabajan en cuestiones estéticas, haciendo cualquier cosa por sobrevivir, sin poder sacar el título de la especialidad homologado" (ESP-H, 43).

Por último, aunque los/as médicos/as no expresan situaciones de discriminación xenofóbica en grado extremo en sus procesos de integración profesional, emergen de los relatos situaciones de cuestionamiento del conocimiento médico por parte de los/as colegas de profesión: "te tratan como si no supieras nada de medicina" (ESP-H, 38). En el caso de los/as pacientes, en ocasiones se han enfrentado a conductas de rechazo hacia los/as médicos/as latinoamericanos/as: "hacen gestos por el solo hecho de que eres extranjero. $Y$ hay que aprender a poder sobrellevar esto e intentar que la atención sea lo más adecuada posible" (R1-H, 31).

\section{Portugal}

Los/as médicos/as que participan en la investigación contratados/as en origen por el Ministerio de Salud se incorporaron laboralmente en el sistema público de salud. Concretamente los/as médicos/as de Cuba, Colombia y de Costa Rica fueron destinados/as para trabajar en el área de atención primaria, debido principalmente a las grandes carencias en este ámbito, y fueron distribuidos geográficamente en distintas regiones del país: Alentejo, Algarve, Centro, Región Metropolitana de Lisboa y las Azores. Por su parte, los/as médicos/as de Uruguay fueron contratados/as para trabajar en el INEM (Instituto Nacional de Emergência Médica) de Lisboa, Porto, Coimbra, Faro y Beja.

En el ámbito de estos acuerdos institucionales, los/as médicos/as reclutados/as entrevistados/as han tenido una serie de facilidades institucionales en el proceso de integración profesional en el sistema nacional de salud con el fin de agilizar este proceso. En primer lugar, el gobierno les facilitó los trámites para la regularización administrativa y de sus familiares. En segundo lugar, recibieron un proceso formativo para una mejor adaptación en sus centros de trabajo cuya duración varió según el área de salud donde iban a trabajar (tres meses en el caso del INEM y 15 días en atención primaria). Y en tercer lugar, y como ya hemos descrito anteriormente, los/as médicos/as al llegar contaban con el reconocimiento del título de Medicina como parte del proceso de selección de contratación en origen, solo necesitando registrarse en la Organización Médica Portuguesa. Sin embargo, los/as médicos/as de Uruguay y Costa Rica tuvieron problemas para registrarse en la organización médica, lo cual ocasionó demoras en la integración profesional en el sistema sanitario, de entre cuatro y nueves meses, de acuerdo con estos/as médicos entrevistados/as y con noticias de la prensa portuguesa sobre los médicos costarricenses.

A pesar de las mayores facilidades institucionales en la integración profesional de estos/as médicos/as por formar parte de un acuerdo entre dos países, las personas entrevistadas relataron haber sufrido situaciones de xenofobia en sus centros de trabajo por parte de algunos/as colegas de trabajo y superiores, manifestadas en desconfianza profesional inicial y actitudes de rechazo y discriminación. Estas actitudes xenófobas, que se manifestaron sobre todo inicialmente, dificultaron en cierto modo los procesos de inserción laboral de estos/as médicos/ as contratados/as en origen:

se nota un poco el trato y la actitud altiva y los argumentos un poco de discriminación: «ustedes son colombianos y nosotros no sabemos qué tipo de educación tienen» (...) Y de hecho, muchos compañeros, enfermeras sí nos comunican que es evidente ese tipo de trato para con nosotros. Entonces sí hay actitudes y acciones discriminatorias (GEN-H, 28).

Por otro lado, la mayoría de los/as médicos/as entrevistados/as que emigraron de forma autónoma o independiente -sin acuerdo entre países- se incorporaron laboralmente en los servicios de urgencias de los hospitales públicos. Así, la "puerta de entrada" al mercado de trabajo en salud para estos/as médicos/as suele ser este espacio laboral de salud, en algunos casos como una etapa transitoria hasta conseguir un mejor empleo, pero lo más usual es que este tipo de actividad termine convirtiéndose en una situación más o menos permanente, en combinación con el desempeño de otro tipo de trabajos.

\section{Conclusiones}

La contratación internacional de médicos/as latinoamericanos/as en España y Portugal presenta similitudes, aunque con ciertas singularidades en cada contexto. Tanto en Portugal como en España la mayoría emigran de forma autónoma e independiente, mientras que el reclutamiento internacional 
en origen sigue siendo más bien minoritario. No obstante, encontramos divergencias en esta última estrategia de contratación en cuanto a los agentes de reclutamiento: mientras que en el caso español las contrataciones en origen se realizan a nivel institucional, es decir, entidades sanitarias concretas (públicas, privadas o mixtas) reclutan médicos/as en el exterior, en Portugal ha sido el Estado, a través del Ministerio de Salud, el principal agente de reclutamiento de los/as médicos/as a través de acuerdos bilaterales firmados con algunos países latinoamericanos.

El reconocimiento de credenciales para la práctica de la Medicina en general involucra los mismos procesos tanto en España como en Portugal: acreditación de títulos académicos y obtención de una licencia en las asociaciones médicas respectivas. Sin embargo, en Portugal existen mayores barreras institucionales para ejercer la profesión médica. Así, mientras que en España uno de los principales obstáculos que enfrentan los/as especialistas formados/as en el extranjero es el reconocimiento de este título, en Portugal los/as médicos/as experimentan dificultades en cada uno de los tres niveles de reconocimiento académico y profesional. Esta situación puede deberse al mayor grado de intervención en la regulación de la profesión médica de las organizaciones profesionales en Portugal en comparación con España. Además, en España los/as médicos/as de América Latina tienen algunas ventajas en el reconocimiento del título de Medicina debido a los acuerdos bilaterales que facilitan dicho proceso y al hecho de compartir el mismo idioma, lo que pone de relieve la importancia de los lazos coloniales históricos y culturales en el reconocimiento de las cualificaciones entre los países.

En el proceso de integración profesional, los/as médicos/as reclutados en origen, tanto en Portugal como España, presentan una serie de facilidades institucionales en cuanto a la regularización administrativa de su estatus legal en el país, la adaptación al nuevo entorno laboral a través de procesos formativos, y el reconocimiento del título de Medicina. Por otro lado, los/as médicos/as que emigran de forma autónoma experimentan un amplio rango de situaciones. En este sentido, la vinculación a un trabajo al migrar de forma autónoma depende de los títulos académicos reconocidos, de las redes profesionales y personales, y de las ofertas en el mercado médico laboral público, privado y mixto. De esta forma, se presentan situaciones que van desde los/as médicos/as latinoamericanos/as más vulnerables que solo tienen la homologación del título de la carrera y el permiso de estudiante, y que ocupan las peores posiciones en el mercado médico laboral principalmente privado, hasta aquellos/as con la nacionalidad y la especialidad hecha o ya homologada en el país de destino, y que han podido tener una trayectoria profesional en instituciones públicas y privadas de prestigio. No obstante, a pesar de estas diferencias encontradas en los dos perfiles, los problemas de discriminación en el ámbito institucional sanitario, tanto por parte de colegas/superiores como por pacientes, afectan indistintamente a ambos grupos y en los dos contextos nacionales.

\section{Notas}

1 Este artículo es resultado de dos investigaciones: la tesis doctoral con título "Cartografías de profesionales de la medicina. Conversaciones con médicas-os latinoamericanas-os desde España" (2012-2015) y el proyecto posdoctoral "Skilled migration of health professionals in Portugal and Spain: a comparative study on the conditions of integration into the host society" (2010-2013), financiado por la Fundação para a Ciência e a Tecnologia de Portugal.

2 Las citas de los extractos de los/as entrevistados/as siguen el criterio: médico/a general, especialista o médico/a residente (GEN, ESP, R1, R2, R3...), género $(H / M)$ y edad.

3 La información relativa a la contratación en origen procede de los informantes-clave y del grupo de médicos/ as contratados/as en origen.

4 La ley se encuentra en: http://www.boe.es/boe/ dias/2010/05/03/pdfs/BOE-A-2010-6960.pdf

5 http://www.gacetamedica.com/gaceta/articulo.aspx?i dart $=752604 \&$ idcat $=797 \&$ tipo $=2$ [consulta diciembre 2013]

Las asociaciones de médicos/as extranjeros/as en España, especialmente ASOMEX, han jugado un papel en el diseño de esta nueva ley al realizar presión sobre las instituciones del sistema sanitario y los partidos políticos.

6 Cita traducida del portugués al español.

\section{Referencias bibliográficas}

COMISIÓN EUROPEA (2010), "Planificación de la fuerza laboral de profesionales de medicina y enfermería en una selección de países europeos: Francia, Alemania, España, Suecia y Reino Unido", en CE (coord.), Migraciones profesionales LA-UE. Oportunidades para el desarrollo compartido, CE.

EUROPEAN OBSERVATORY ON HEALTH SYSTEMS AND POLICIES (EOHSP) (2006), The Health Care Workforce in Europe: Learning from Experience, WHO, Trowbridge, Wilts, London, The Cromwell Press.

MASANET, E. (2012), "A imigração qualificada dos(as) médicos(as) brasileiros(as) na Espanha e em Portugal: quais as diferenças e as semelhanças?", in B. Padilla et al. (org.), Novas e velhas configurações da imigração brasileira na Europa. Atas do 2.0 Seminário de Estudos sobre a Imigração Brasileira na Europa, Lisboa, ISCTE, pp. 159-174.

MINISTÉRIO DA SAÚDE (2015), Recursos Humanos Estrangeiros no Ministério da Saúde - Actualizaçao 2011-2014, Lisboa, Administração Central do Sistema de Saúde, IP.

MINISTERIO DE SANIDAD, POLÍTICA SOCIAL E IGUALDAD (MSSSI) y UNIVERSIDAD DE LAS PALMAS DE GRAN CANARIA (UPGC) (2011), Oferta y necesidad 
de especialistas médicos en España (2010-2025), MSSSI, Madrid.

ORGANIZACIÓN PARA LA COOPERACIÓN Y EL DESARROLLO ECONÓMICO (OCDE) (2008), The Looming Crisis in the Health Workforce: How Can OECD Countries Respond?, OCDE.

RIBEIRO, J. et al. (2013), "Health professionals moving to and out of Portugal: a typical case?", Health Policy, 114 (2-3), pp. 97-108.
RUSSO, G. et al. (2012), "What influences national and foreign physicians' geographical distribution? An analysis of medical doctors' residence location in Portugal", BMC Human Resources for Health, 10 (12), pp. 1-11.

SEVILLANO, E. (2014), "No hay plaza para tanto médico" en El País, 30 de enero de 2014, http:// sociedad.elpais.com/sociedad/2014/01/30/actualidad/1391113575_604573.html.

Submetido a 05/10/2015. Aceite para publicação a 05/12/2015.

María Cristina Romero (mariacristina.romero@cps.ucm.es). Universidad Complutense de Madrid, Facultad de Ciencias Políticas y Sociología, Departamento de Sociología I. Campus de Somosaguas, 28223 Pozuelo de Alarcón, Madrid, España.

Erika Masanet (erika.masanet@uv.es). Universidad de Valencia, Facultad de Ciencias Sociales, Departamento de Sociología y Antropología Social. Av. Tarongers, 4b, 46021 València, España. 